\title{
Methods for the determination of the effective heating radius in district heating systems taking into account reliability
}

\author{
Ekaterina Mednikova ${ }^{1}$, Ivan Postnikov ${ }^{1, *}$ \\ ${ }^{1}$ Melentiev Energy Systems Institute of Siberian Branch of the Russian Academy of Sciences, Laboratory of Heat Supply Systems, \\ 664033 Irkutsk, Russia
}

\begin{abstract}
Methods for determining the effective heating radius (EHR) in district heating systems (DHS) are developed, taking into account ensuring of reliable heat supply to consumers. EHR is such a length (distance) of a heat network $(\mathrm{HN})$ from a district heat source (HS) to a consumer, within which the district heat supply of a given consumer is economically more profitable than a distributed (autonomic) one. Thus, the search for EHR for different transmission heat pipelines (TP) connected to the considered HS determines of its operation zones in different districts of the city. In addition, to the main problem of the EHR search, the assessment of nodal reliability indices (RI) for each consumer is carried out, on the basis of which the obtained EHR solutions are corrected (if necessary). As a result, we can determine the zones of district heating in DHS, within the framework of which not only energy and economically effective, but also reliable heat supply to consumers is provided. The developed methodical ensuring was used in the development of an optimal heat supply scheme for the Irkutsk city (Eastern Siberia, Russia). The brief review on the results of the conducted practical researches case study is presented.
\end{abstract}

\section{Introduction}

The effectiveness of the development and ensuring of benefits of district heating is mainly connected with the optimal planning of the functioning and modernization of district heating systems (DHS), which is the determination of their scale, the distance of heat carrier transportation, requirements for the quality and reliability of heating to consumers, taking into account the increasing demand for thermal energy.

The validity of the decisions made on these issues is largely determined by the heating radius or the length (distance) of the heat network (HN) from district heat source (HS) to the consumer. Thus, the effective heating radius (EHR) in DHS is such a length (distance) of a heat network (HN) from a district heat source (HS) to a consumer, within which the district heat supply of a given consumer is economically more profitable than a distributed (autonomic) one. In this regard, the problem of search EHR is one of the key one of designing the effective DHSs of 4th generation [1-3].

The requirements for necessity of solving this problem (EHR determine) are presented in normative documents, for example $[4,5]$. At the same time, at present there is no regulatory document that would contain a detailed and scientifically grounded methodology for calculating the EHR. Thus, the problem of determining the optimal scale of development of DHS based on the index of EHR, that is the one of the key point in the efficiency heat supply scheme, requires the development of new scientifically founded methodological support for it solution.
The analysis of the scientific and technical literature sources on the studied subject showed that, despite the absence of methods in normative documents, the issue of calculating the EHR was given a lot of attention, starting from the time of the formation of district heating [611].The research results obtained in these studies showed that EHR and optimal heat supply areas from HS significantly depend on the structure and parameters of considered DHS. However, the most of proposed methods are aimed at determining a integrated value of EHR for the entire system, assuming that the heat loads are evenly distributed on the territory. Such conditions are met extremely rarely for actual DHSs; therefore, the existing methods for determining EHR are inapplicable for most existing systems. Moreover, the considered methods do not take into account the reliability requirements when calculating EHR, which when implementing the solutions obtained in this way in practice can lead to frequent interruptions of consumers located in EHR area.

\section{Methods}

The main idea of the proposed methodological approach to the determination of EHR is it is necessary to calculate value of EHR for each transmission pipeline (TP) of HN connected to the each district HS in considered DHS. And for consumers located in the EHR zone, the required (standard) level of reliability of heating must be ensured.

The proposed methodical for determining EHR for DHS, taking into account the reliability requirements, is the following main stages.

*Corresponding author: postnikov@,isem.irk.ru 
1. Formation of the initial data for studied DHS: existing zones of district heating, consumer heat loads, locations, technical and economic parameters of HS and $\mathrm{HN}$, reliability parameters of its components (failure and restoration rates).

2. Calculation of flow distribution in district $\mathrm{HN}$ that it is necessary for constrained of acceptable hydraulic modes and determine the level of heat supply to each consumer in different system states (in case of failures in system components). The flow distribution is calculated using the mathematical model (in matrix form) developed within the theory of hydraulic circuits (THC) [12]:

$$
\begin{aligned}
& \mathbf{A x}=\mathbf{g}, \\
& \overline{\mathbf{A}^{\prime}} \mathbf{p}=\mathbf{h}-\mathbf{H}, \\
& \mathbf{h}=\mathbf{S X}|\mathbf{x}|,
\end{aligned}
$$

where A - incidence matrix of linearly independent nodes of HN (for this model other indices are also related to network); $\mathbf{x}$ - vector of flow rates on sections, $\mathrm{t} / \mathrm{h} ; \mathbf{g}$ - vector of flow rates at nodes, $\mathrm{t} / \mathrm{h} ; \overline{\mathbf{A}^{\prime}}$ - transpose of matrix $\overline{\mathbf{A}}(\overline{\mathbf{A}}$ - total incidence matrix of nodes and sections); $\mathbf{p}$ - total vector of pressures at nodes, $\mathrm{Pa} ; \mathbf{h}-$ vectors of head losses in the branches, $\mathrm{Pa} ; \mathbf{H}$ - vectors of operating heads in the branches, $\mathrm{Pa} ; \mathbf{S}$ - diagonal matrix of hydraulic resistances of sections, $\mathrm{Pa} \times \mathrm{h}^{2} / \mathrm{t}^{2} ; \quad \mathbf{X}-$ absolute values of flow rates on branches, $t / h$.

3. Calculation of specific costs for the production and transportation of thermal energy (cost price) for each node of the each TP from the $i$-th HS:

$$
\begin{aligned}
Z_{m}= & c_{i}^{\mathrm{h}}+\alpha k_{i}+(f+\alpha)\left(1 / Q_{i}^{m}\right) \sum_{n \in N(m)}\left(k_{n} d_{n} l_{n}\right)+ \\
& +c_{\mathrm{e}} \tau_{\mathrm{p}}\left(1 / 367 \eta Q_{i}^{m}\right) \sum_{n \in N(m)}\left(\psi_{n} x_{n} l_{n}\right),
\end{aligned}
$$

where $i$ - number of district HS ( $i \in I$, where $I-$ complete set of sources in the considered system); $m$ number of $\mathrm{HN}$ nodes ( $m \in M$, where $M$ - complete set of nodes); $n$ - number of HN sections ( $n \in N$, where $M$ - complete set of sections); $N(m)$ - subset of HN sections from district HS to node $m ; c_{i}^{\mathrm{h}}$ - heat production cost of the $i$-th HS, eur/MWh; $\alpha$ - annuity rate; $k_{i}-$ specific capital investment in heat capacity expansion for $i$-th HS, eur/MW; $f$ - share of HN depreciation, repair and maintenance charges; $Q_{i}^{m}-$ heat load at HN nodal $m$ (consumers if in this nodal thermal energy is consumed), MW; $k_{n}$ - specific investment cost for $n$-th HN section (re)construction, eur/m; $d_{n}$ - diameter of $n$-th $\mathrm{HN}$ section, $\mathrm{m} ; l_{n}-$ length of $n$-th $\mathrm{HN}$ section, $\mathrm{m} ; c_{\mathrm{e}}-$ electricity cost, eur/MWh; $\tau_{\mathrm{p}}$ - time of pump operation under design load, $\mathrm{h} ; \eta$ - pump efficiency; $\psi_{n}$ - specific pressure drop in $n$-th section, $\mathrm{Pa} / \mathrm{m} ; x_{n}$ - heat carrier flow rates in $n$-th network section, $\mathrm{t} / \mathrm{h}$.
4. Assessment of the system-average level of specific costs for the production and transportation of thermal energy:

$$
\bar{Z}=\left(1 / \sum_{i \in I} Q_{i}\right) \sum_{i \in I} Q_{i} Z_{i},
$$

where $Q_{i}$ - connected heat load of the $i$-th district HS, MW; $Z_{i}-$ specific costs for the production and transportation of thermal energy (cost price) for $i$-th HS, eur/MWh (heat).

5. Determination of EHR for each TP from each HS, i.e. subsets of the nodes of this pipeline, for which the specific cost of thermal energy does not not exceed its average level in the system. A node's belonging to EHR zone is formalized according to the following condition:

$$
\begin{gathered}
m \notin M_{\text {eff }}, \text { if } Z_{m} \geq \bar{Z}, \\
m \in M_{\text {eff }}, \text { if } Z_{m}<\bar{Z},
\end{gathered}
$$

where $M_{\text {eff }}$ - subset of nodes located in EHR zone.

6. Determination of two main reliability indices (RI) the availability factor (AF) and the failure-free operation probability (FOP) [13]. These indices can be calculated for each node according to the methodology for reliability assessing for DHS, presented in [13], by following formulas:

$$
\begin{gathered}
K_{m}=\sum_{s \in E} p_{s}\left[\left(\tau_{0}-\tau_{s m}\right) / \tau_{0}\right], \\
R_{m}=\exp \left(-p_{0} \sum_{s \in E} \sum_{n \in N_{s}} \tau_{s m} \lambda_{n}\right),
\end{gathered}
$$

where $K_{m}$ and $R_{m}$ - nodal AF and FOP respectively; $s$ - number of system state; $E$ - complete set of system states; $p_{s}$ - probability of system state $s ; \tau_{0}$-duration of the heating period (or other designing period of time), $\mathrm{h} ; \tau_{s m}$ - part of the heating period within which state $s$ is a failed state for node $m ; p_{0}$ - probability of totally operable system state $(s=0) ; N_{s}$ - subset of $\mathrm{HN}$ sections failure of which corresponds to transit to some state $s ; \lambda_{n}$ - failure rate of $\mathrm{HN}$ sections $n, 1 / \mathrm{h}$.

Probabilities of system states is determined by solving the equation system of the stationary markov process describing the evolution of events in the system. Applied of markov models for the solving of reliability problems in DHS in more detail is considered in papers [13-16].

7. Verification of solutions for EHR to meet the requirements of the reliability of heat supply is carried out in relation to the normative values of nodal RI. Thus, for each node belonging to EHR zone, the following conditions must be met:

$$
\begin{aligned}
& K_{m} \geq K_{0}, m \in M_{\mathrm{eff}}, \\
& R_{m} \geq R_{0}, m \in M_{\mathrm{eff}},
\end{aligned}
$$


where $K_{0}$ and $R_{0}$ - standard values of $\mathrm{AF}$ and FOP, respectively. If conditions (10) and (11) are violated, EHR zone is corrected to limited to the node that is most distant from HS, but in which the reliability requirements are met. If EHR zone cannot be reduced (for example, if there are no other HS to which consumers can be connected that go beyond the boundaries of this zone due to reliability conditions), then measures are developed to increase reliability: using redundant pipelines, more reliable components, replacement of worn out HN sections. After that, a return to point 3 of described methodical is carried out. At the same time, it is necessary to take into account additional investments for measures to improve reliability when calculating the specific annual costs for the transportation of thermal energy.

\section{Brief report on case study}

According to the presented methodology, EHR was calculated for the DHS of the Irkutsk city (Eastern Siberia, Russia). Thermal energy for district heating is produced at the Novo-Irkutskaya CHPP (NICHPP), the installed heat capacity of which is $2010 \mathrm{MW}$ and the electric power 708 MW. An scheme of the DHS on Irkutsk city map is presented in the Fig. 1.

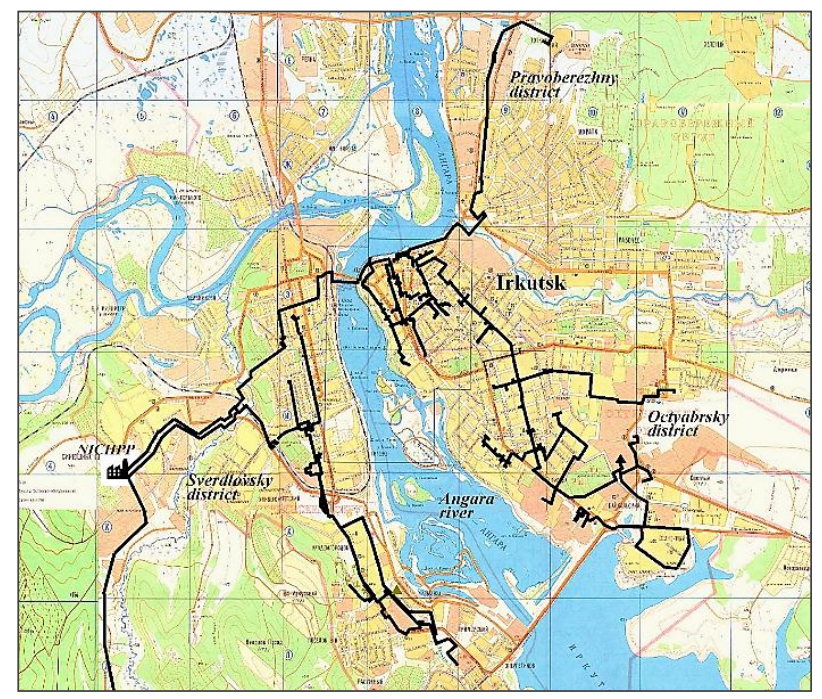

Fig. 1. DHS of Irkutsk on the city map

The total length of HN from NICHPP makes up 474.3 $\mathrm{km}$, including $112.9 \mathrm{~km}$ of four TP directions for different district of the city: TP-1, TP-2, TP-3 and TP-4.

According to developed methods, EHR was calculated for the NICHPP in Irkutsk. The GAMS (General Algebraic Modeling Systems) software package is used as a solver. An aggregated calculated scheme of the considered DHS with decisions on EHR is presented in the Fig. 2. Its values for the NICHPP (disregarding reliability requirements) along $\mathrm{TP}$ and their branches are limited by nodes highlighted in red in the Fig. 2. The values of the radius vary from $2 \mathrm{~km}$ to $15.5 \mathrm{~km}$. Analysis of heating system reliability shows that the standard values of AF and FOP, set according to [13], are not met for some nodes that belong to EHR zones.

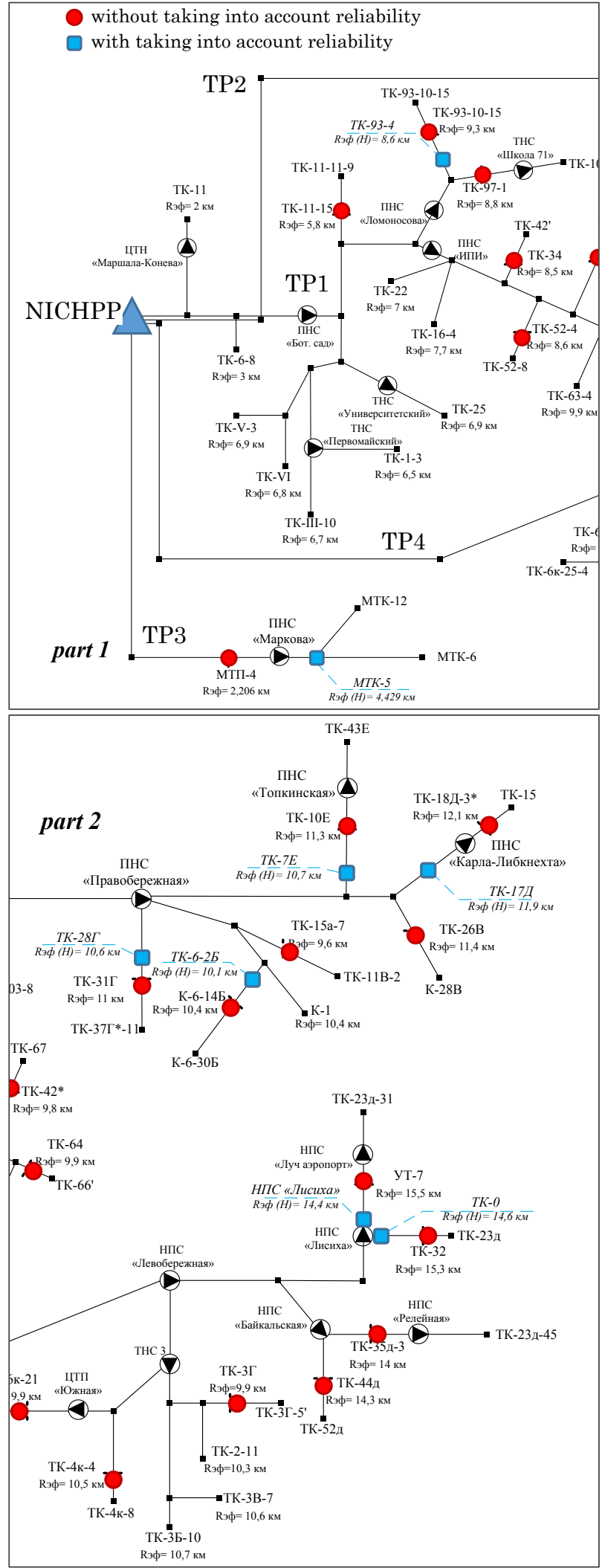

Fig. 2. Aggregate scheme of the DHS with decisions on EHR

According to RI obtained, we adjusted EHR. In the Fig. 2, the boundaries of EHR taking into account reliability, are shown in blue colour. Thus, the maximum EHR for NICHPP will decline from $15.5 \mathrm{~km}$ to $14.6 \mathrm{~km}$, and the total length of the heat transmission networks in EHR zone will shrink by $4.2 \mathrm{~km}$ and make up $67.7 \mathrm{~km}$. 
According to the obtained results, considering reliability requirements, the zone of EHR embraces 284.3 $\mathrm{km}$ of $\mathrm{HN}$ out of $474.3 \mathrm{~km}$, i.e. $40 \%$ of the networks are beyond EHR zone.

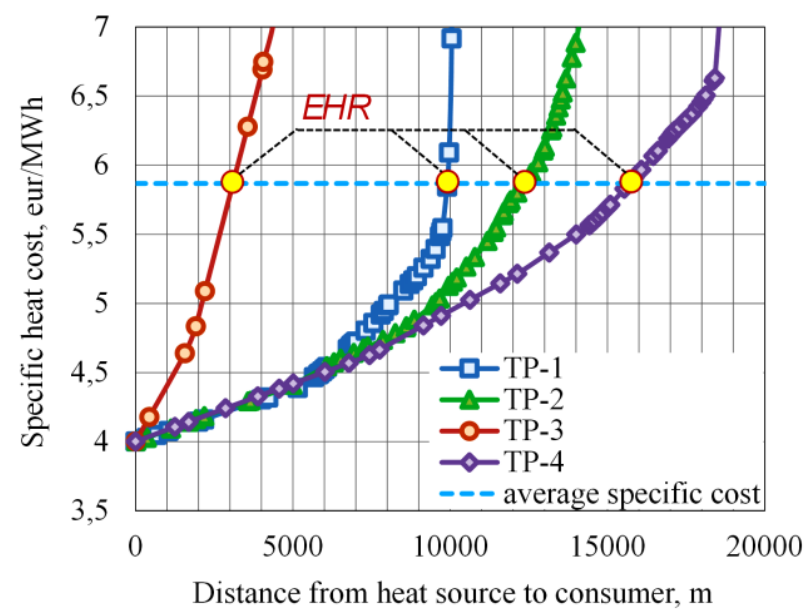

Fig. 3. Specific heat cost for the longest branches of TPs of DHS in Irkutsk city on based NICHPP: crossing of line with average level (dashed line) corresponds to decision on EHR

Specific heat cost for the longest branches of TP and average specific heat cost for the system are presented in the Fig. 3. Correlation between the heat load and a material characteristic of the heat network has a significant influence on the specific heat cost for each node [10]. The greater the specific material characteristic of pipeline (per heat load unit), the higher the specific heat cost and vice versa.

\section{Conclusions}

The paper proposes a methodology for the calculation of EHR for DHS. The radius is determined as a set of nodes in $\mathrm{HN}$ scheme. The cost of heat production and transportation at these nodes does not exceed its average values throughout the considered DHS. The methodology also provides for the requirements for the reliability of heating to consumers, which is assessed using nodal RI. The decision of connecting new consumers within the boundaries of EHR requires separate assessments using an additional criterion - the local EHR.

The carried out practical calculation (case study) on the actual DHS showed that the methodological approach to calculating EHR for the network nodes for each TP from district HS allows obtaining the most reasonable and detailed estimates of the efficiency of heat supply to consumers.

Proposed methods are universal for any type and scale of DHS. It takes into account individual features of territories and can be successfully applied in design of heat supply schemes.

The reported study was funded by RFBR, project number 20-08-00488 A

\section{References}

1. H. Lund, P. Alberg Østergaard, M. Chang M et al., Energy 164 (2018)

2. H. Lund, S. Werner, R. Wiltshire et al., Energy 38 (2014)

3. H. Lund, N. Duic, P. Østergaard, B. Mathiesen, Energy 110 (2016)

4. Russian Federal law of 27.07.2010 No.190-FL (edit. of 29.07.2017), On heat supply (Moscow, 2017)

5. Order of the Ministry of the Regional Development of the RF of 26.05.2011 No.244, On approval of the methodical recommendations for the development of draft general plans for settlements and urban districts (Moscow, 2011)

6. L. Yakimov, Heat and power 9 (1931)

7. V. Khasilev, Issues of technical and economic calculation of heating networks (State Energoizdat, Moscow, 1957)

8. L. Khrilev, I. Smirnov, Optimization of cogeneration and district heating systems (Energy Pub., Moscow, 1978)

9. V. Papushkin, Heat supply news 9 (2010)

10. V. Stennikov, E. Iakimetc, Energy 110 (2016)

11. V. Stennikov, E. Mednikova, I. Postnikov, A. Penkovskii, Environmental and Climate Technologies, 23, 2 (2019)

12. A. Merenkov, V. Khasilev, Theory of hydraulic circuits (Nauka, Moscow, 1985)

13. E. Sennova, A. Smirnov, A. Ionin et al., Reliability of heat supply systems (Nauka, Novosibirsk, 2000)

14. I. Postnikov, V. Stennikov, E. Mednikova, A. Penkovskii, Applied Energy 227 (2018)

15. V. Stennikov, I. Postnikov, Power Technology and Engineering 47, 6 (2014)

16. I. Postnikov, V. Stennikov, Energy Reports 6, 2 (2020) 